ARTICLE

https://doi.org/10.1038/s41467-019-11840-3

\title{
Asymmetric catalysis mediated by a mirror symmetry-broken helical nanoribbon
}

\author{
Zhaocun Shen (10) 1,2,3,7, Yutao Sang (10 1,3,7, Tianyu Wang (10 1,7, Jian Jiang ${ }^{4}$, Yan Meng 1,3, Yuqian Jiang ${ }^{4}$, \\ Kou Okuro (iD 2 ${ }^{2}$, Takuzo Aida (1D) ${ }^{2,5}$ \& Minghua Liu (10) ${ }^{1,3,4,6}$
}

\begin{abstract}
Although chirality has been recognized as an essential entity for life, it still remains a big mystery how the homochirality in nature emerged in essential biomolecules. Certain achiral motifs are known to assemble into chiral nanostructures. In rare cases, their absolute geometries are enantiomerically biased by mirror symmetry breaking. Here we report the first example of asymmetric catalysis by using a mirror symmetry-broken helical nanoribbon as the ligand. We obtain this helical nanoribbon from a benzoic acid appended achiral benzene-1,3,5-tricarboxamide by its helical supramolecular assembly and employ it for the $\mathrm{Cu}^{2+}$-catalyzed Diels-Alder reaction. By thorough optimization of the reaction (conversion: $>99 \%$, turnover number: 90 ), the enantiomeric excess eventually reaches $46 \%$ (major/ minor enantiomers $=73 / 27$ ). We also confirm that the helical nanoribbon indeed carries helically twisted binding sites for $\mathrm{Cu}^{2+}$. Our achievement may provide the fundamental breakthrough for producing optically active molecules from a mixture of totally achiral motifs.
\end{abstract}

\footnotetext{
${ }^{1}$ Beijing National Laboratory for Molecular Sciences, CAS Key Laboratory of Colloid, Interface and Chemical Thermodynamics, CAS Research/Education Center for Excellence in Molecular Sciences, Institute of Chemistry, Chinese Academy of Sciences, Beijing 100190, P. R. China. ${ }^{2}$ Department of Chemistry and Biotechnology, School of Engineering, The University of Tokyo, 7-3-1 Hongo, Bunkyo-ku, Tokyo 113-8656, Japan. ${ }^{3}$ University of Chinese Academy of Sciences, Beijing 100049, P. R. China. ${ }^{4}$ CAS Center for Excellence in Nanoscience, National Center for Nanoscience and Technology, Beijing 100190, P. R. China. ${ }^{5}$ RIKEN Center for Emergent Matter Science, 2-1 Hirosawa, Wako, Saitama 351-0198, Japan. ${ }^{6}$ Collaborative Innovation Center of Chemical Science and Engineering, Tianjin 300072, P. R. China. ${ }^{7}$ These authors contributed equally: Zhaocun Shen, Yutao Sang, Tianyu Wang. Correspondence and requests for materials should be addressed to Z.S. (email: shenzc@iccas.ac.cn) or to K.O. (email: okuro@macro.t.u-tokyo.ac.jp) or to T.A. (email: aida@macro.t.u-tokyo.ac.jp) or to M.L. (email: liumh@iccas.ac.cn)
} 
C hirality has been recognized as an essential entity for life. However, it still remains a big mystery how the homochirality in nature emerged in essential biomolecules, such as sugars and amino acids ${ }^{1,2}$. Certain achiral molecules are known to form crystals adopting a chiral geometry, which usually form as a conglomerate, i.e., a racemic mixture of enantiomorphic crystals. Post chemical transformation of such geometrically chiral single crystals may afford enantiomerically enriched products $^{3-5}$. This is one of the typical approaches to absolute asymmetric synthesis ${ }^{6-8}$, which has attracted long-term attention since the time of Pasteur. If mirror symmetry breaking occurs in the crystallization of achiral compounds, a mixture of unequal numbers of enantiomorphic crystals forms. For example, Kondepudi et al. reported that stirred crystallization is often effective to induce mirror symmetry breaking, leading to a high enantiomeric excess $(e e)$ of the resulting crystal $^{9-11}$. Notably, Viedma et al. later demonstrated that continuous grinding of a slurry of a racemic mixture of enantiomorphic crystals could cause its deracemization to produce an enantiopure product (Viedma ripening $)^{12-18}$. Especially, Blackmond and coworkers for the first time experimentally supported a hypothesis of the emergence of solid-phase homochirality from racemic conglomerates composed of rapidly racemizing enantiomers ${ }^{13,19,20}$. Tsogoeva and coworkers achieved a complete deracemization $(100 \% e e)$ of an asymmetric reaction product by combining its reversible asymmetric reaction and physical grinding of crystals involved ${ }^{21,22}$. Nevertheless, an even more challenging subject to tackle is how one can amplify the enantiomeric bias generated as a consequence of mirror symmetry breaking. In 2008, Soai and coworkers employed such a mirror symmetry-broken crystalline mixture of cytosine as a chiral auxiliary for the enantioselective addition reaction between diisopropylzinc and pyrimidine-5carbaldehyde ${ }^{23}$. Although the range of eligible substrates is highly limited, this reaction is well-known for amplifying the enantiomeric bias in an autocatalytic manner ${ }^{24}$.

Some covalent helical polymers ${ }^{25-35}$ and their noncovalent analogues ${ }^{36-40}$, prepared from certain chiral monomers, are known to serve as chiral ligands for asymmetric catalysis. Suginome and coworkers have developed a series of helical poly(quinoxaline-2,3diyl) copolymers bearing metal-binding phosphine pendants as chiral ligands for several palladium-catalyzed asymmetric reactions ${ }^{29-34}$. Examples of noncovalent helical supramolecular assemblies include hydrogen-bonded helical stacks of chiral benzene-1,3,5-tricarboxamide (BTA) derivatives ${ }^{37-40}$. In our previous paper, we reported that an achiral BTA motif that was appended with an ethyl cinnamate group in each of its three side chains selfassembled into helical nanoribbons, where mirror symmetry breaking occurred under certain conditions to form a mixture of unequal numbers of right-handed $(P)$ and left-handed $(M)$ helical nanoribbons ${ }^{41,42}$. We envisioned that such mirror symmetrybroken supramolecular assemblies ${ }^{41-54}$ might mediate asymmetric reactions if they are properly designed to accommodate catalytically active transition metal ions.

Herein, we report the first example of asymmetric catalysis realized by using a mirror symmetry-broken helical supramolecular assembly of an achiral BTA motif as the ligand (Fig. 1). The achiral BTA derivative $\left(\mathbf{B T A}^{\mathbf{B A}}\right.$, Fig. 1a) ${ }^{55}$ carries a benzoic acid group in each of its three arms for chelating a $\mathrm{Cu}^{2+}$ ion that can catalyze the Diels-Alder cycloaddition reaction between aza-chalcone and cyclopentadiene (Fig. 1c). The enantiomeric excess of the reaction reaches $46 \%$ (conversion: $>99 \%$, turnover number: $\sim 90)$.

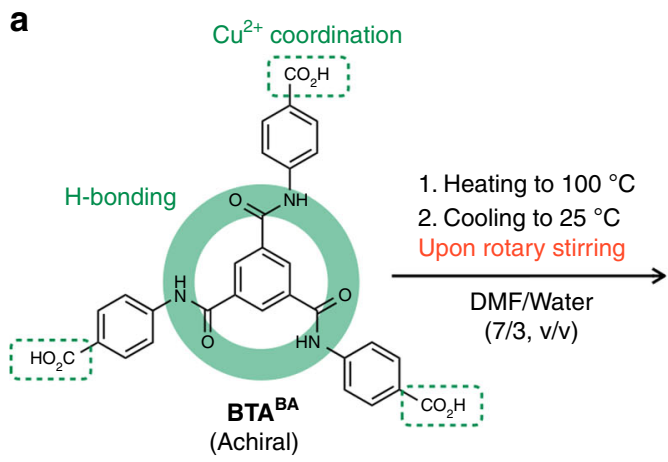

b

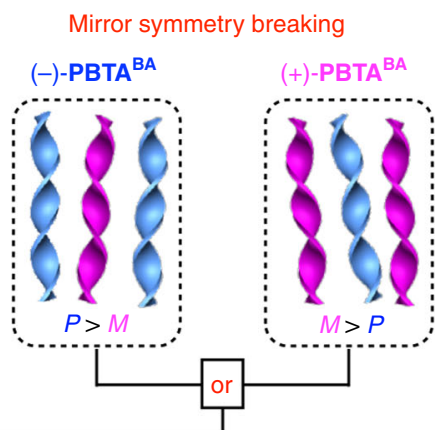

C

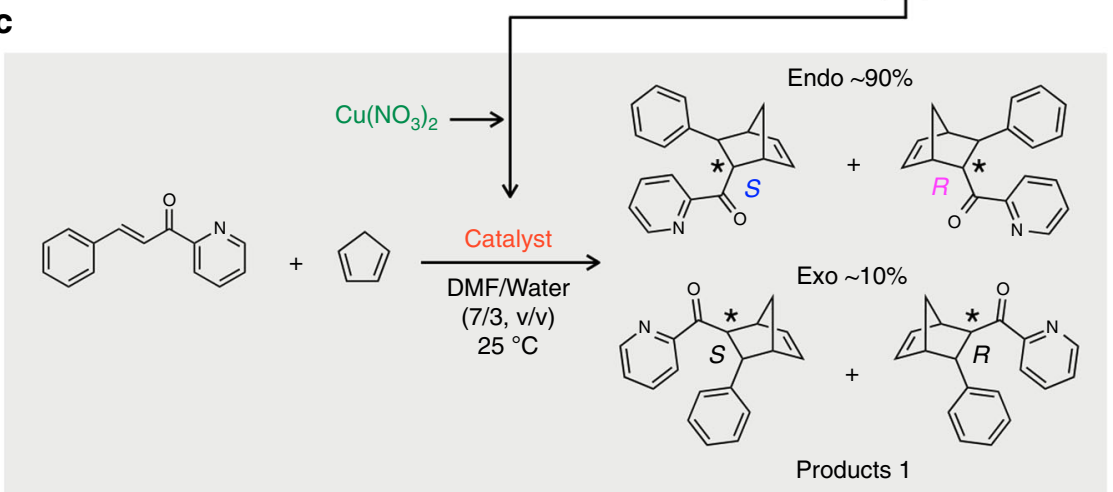

Fig. 1 Mirror symmetry breaking of helical supramolecular nanoribbons for asymmetric catalysis. $\mathbf{a}$, b Molecular structure of the achiral BTABA monomer and schematic representation of the preparation of helical $\mathbf{P B T A} \mathbf{B A}^{\mathbf{B A}}$ nanoribbons with either $(P)$ - or $(M)$-dominant helical handedness by applying rotary stirring during the cooling process of a DMF/water solution of $\mathbf{B T A}^{\mathbf{B A}}$. $(P)$ - and $(M)$-dominant $\mathbf{P B T} \mathbf{A}^{\mathbf{B A}}$ display negative $\left((-)-\mathbf{P B T A} \mathbf{B A}^{\mathbf{B A}}\right)$ and positive $\left((+)-\right.$ PBTA $\left.^{\mathbf{B A}}\right)$ CD signs at $316 \mathrm{~nm}$, respectively. $\mathbf{c}$ Diels-Alder cycloaddition reaction between aza-chalcone and cyclopentadiene catalyzed by $\mathrm{Cu}^{2+}$ in combination with (-)-PBTA $\mathbf{A}^{\mathbf{B A}}$ or $(+)-\mathbf{P B T A}^{\mathbf{B A}}$. The reaction affords the endo isomer preferentially $(\sim 90 \%)$. The $(S)$-endo and $(R)$-endo enantiomers were obtained preferentially when (-)-PBTA $\mathbf{A}^{\mathbf{B A}}$ and $(+)-\mathbf{P B T A}^{\mathbf{B A}}$ were used, respectively 


\section{Results}

Mirror symmetry breaking of helical nanoribbons. BTA ${ }^{\mathbf{B A}}$ was synthesized and unambiguously characterized (see Supplementary Methods, Supplementary Figs. 1-6). Analogous to previous examples $^{41,42,55}$, BTA $^{\mathbf{B A}}$ self-assembled into $\mathbf{P B T A}^{\mathbf{B A}}$, a supramolecular helical nanoribbon (Fig. 1b). We found that this selfassembly event proceeds with mirror symmetry breaking to afford either $(P)$-dominant PBTA $^{\mathbf{B A}}\left((-)\right.$-PBTA $\left.^{\mathbf{B A}}\right)$ or $(M)$-dominant PBTA $^{\mathbf{B A}}\left((+)\right.$-PBTA $\left.{ }^{\mathbf{B A}}\right)$ (Fig. 1b) under physical agitation generated by magnetic rotary stirring. The method for the preparation of mirror symmetry-broken PBTA $^{\mathbf{B A}}$ is slightly complicated as described below (Fig. 2) but quite analogous to that reported in our previous paper ${ }^{2}$ : Water $(0.3 \mathrm{~mL})$ was added to a $N, N$ dimethylformamide (DMF, $0.7 \mathrm{~mL}$ ) solution of $\mathbf{B T A}^{\mathbf{B A}}(3 \mathrm{mg}$, $5.3 \times 10^{-6} \mathrm{~mol}$ ) in a 5 -mL cylindrical glass vial (Fig. $2 \mathrm{a}$ ), whereupon the mixture, when allowed to stand at $25^{\circ} \mathrm{C}$ for $2 \mathrm{~h}$, underwent physical gelation (Fig. $2 \mathrm{~b}$ and Supplementary Tables 1 and 2). The gel was heated at $100{ }^{\circ} \mathrm{C}$ for $3 \mathrm{~min}$, and the resultant homogeneous solution (Fig. 2c) was allowed to cool to $25^{\circ} \mathrm{C}$ with magnetic rotary stirring (1200 rpm) for $40 \mathrm{~min}$, yielding a white suspension (Fig. $2 \mathrm{~d}$, e), which turned out to be optically active (see below). This result was highly reproducible (Supplementary Fig. 7), except that the sign of the circular dichroism (CD) activity at $316 \mathrm{~nm}$ of the resultant suspension was either negative $\left((-)-\mathbf{P B T A}^{\mathbf{B A}}\right)$ or positive $\left((+)-\right.$ PBTA $\left.^{\mathbf{B A}}\right)$ stochastically (see below). Although the CD sign was independent of the direction of rotary stirring (Supplementary Fig. 7), a stable CD activity, once emerged, lasted without fading unless the suspension was heated to transform into a homogeneous solution. Note that, as demonstrated for the suspension of (-)-PBTA ${ }^{\text {BA }}$ in Supplementary Fig. 8, its CD spectrum was barely contaminated with a spectral artifact due to linear dichroism (LD). Next, an aliquot of the suspension was taken out from the glass vial and cast on a silicon wafer for air-drying, followed by evacuation under reduced pressure. Scanning electron microscopy (SEM) of the resulting specimens revealed that (-)-PBTA ${ }^{\mathbf{B A}}$ consisted mostly of $(P)$-helical nanoribbons (Fig. 2d), whereas $(M)$-helical nanoribbons were dominant in (+)-PBTA ${ }^{\mathbf{B A}}$ (Fig. 2e). The gel (Fig. 2b) was CD-silent (Supplementary Figs. 9 and 10). Consistently, its cross-linked 3D network appeared to be composed of equal numbers of $(P)$ - and $(M)$-helical nanoribbons (Fig. 2b). The same held true when a clear, hot solution of BTA $^{\mathbf{B A}}$ (Fig. 2c) was allowed to cool to $25^{\circ} \mathrm{C}$ without rotary stirring (Fig. 2f and Supplementary Fig. 11). Not only magnetic rotary stirring but also mechanical rotary stirring using an overhead stirrer, vortex mixing, and even sonication resulted in the formation of a CD-active suspension of (-)-PBTA ${ }^{\mathbf{B A}}$ or (+)-PBTA ${ }^{\mathbf{B A}}$ (Supplementary Fig. 12).

Interestingly, an ethyl ester derivative of $\mathbf{B T A}^{\mathbf{B A}}$ did not assemble into helical nanoribbons under otherwise identical conditions to those for $\mathbf{B T A}^{\mathbf{B A}}$ (Supplementary Fig. 13), suggesting that the peripheral carboxylic acid groups of $\mathbf{B T A} \mathbf{B A}^{\mathbf{B A}}$ play a critical role in its helical assembly. Fourier transform infrared (FT-IR) spectroscopy of a xerogel of PBTA ${ }^{\mathbf{B A}}$, dried under reduced pressure, showed small vibrational bands at 2665 and $2543 \mathrm{~cm}^{-1}$ (Supplementary Fig. 14), which are characteristic of the $\mathrm{OH}$ stretching vibrations due to hydrogen-bonded dimers of carboxylic acids. Two bands at 3311 and $1654 \mathrm{~cm}^{-1}$, assignable

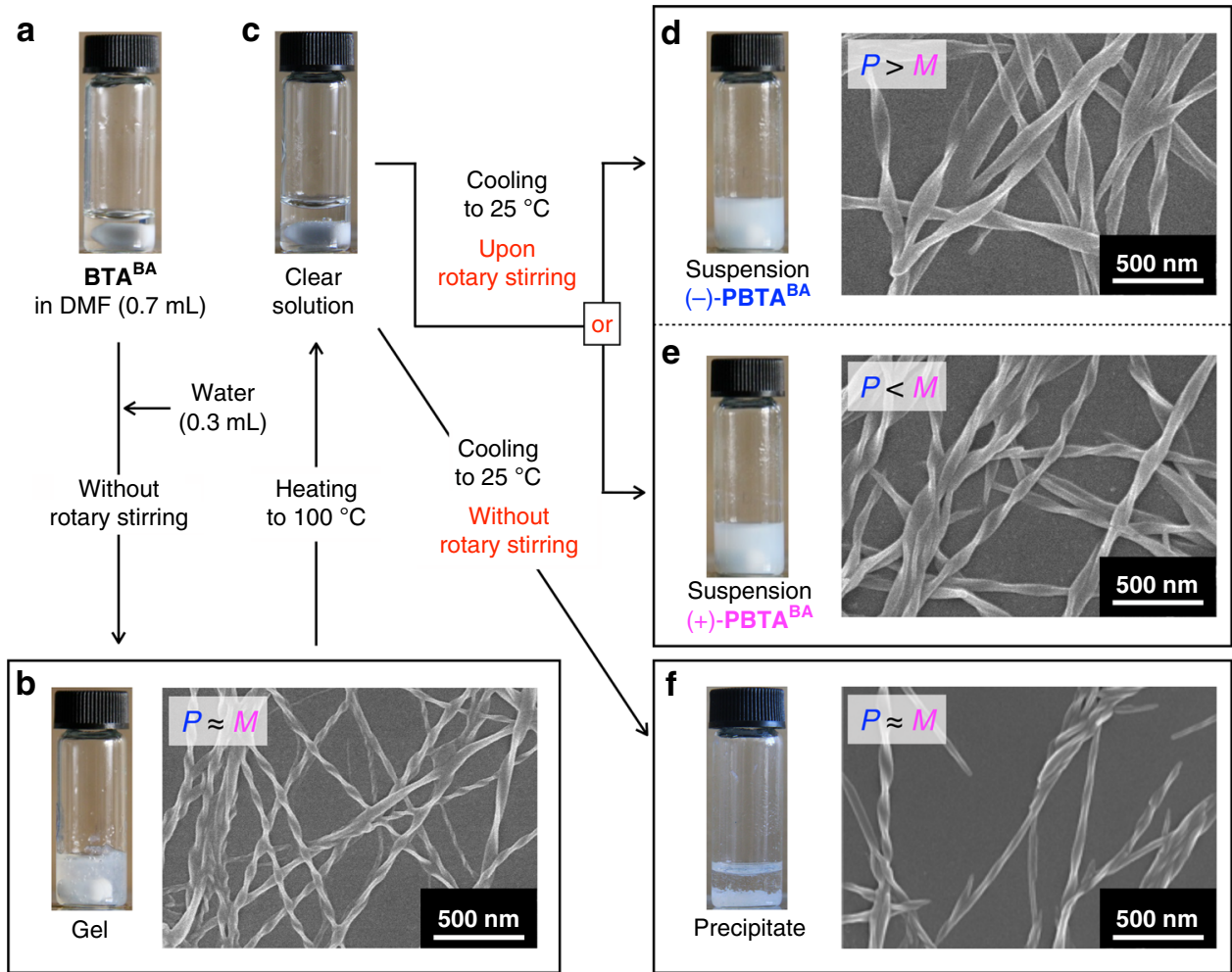

Fig. 2 Procedures for the preparation of mirror symmetry-broken helical PBTABA nanoribbons. a Photograph of a DMF $(0.7 \mathrm{~mL})$ solution of $\mathbf{B T A}$ BA $(3 \mathrm{mg}$, $\left.5.3 \times 10^{-6} \mathrm{~mol}\right)$. b Photograph of a CD-silent gel of $\mathbf{P B T A}^{\mathbf{B A}}\left(\left[\mathbf{B T A}^{\mathbf{B A}}\right]=5.3 \mathrm{mM}\right)$ formed in DMF/water $(7 / 3, \mathrm{v} / \mathrm{v} ; 1 \mathrm{~mL})$ and SEM image of its air-dried specimen. The gel can be prepared via an antisolvent method through direct addition of water (poor solvent) into a DMF (good solvent) solution of BTABA without magnetic rotary stirring. c Photograph of a DMF/water ( $7 / 3, \mathrm{v} / \mathrm{v} ; 1 \mathrm{~mL})$ solution of $\mathbf{B T A}^{\mathbf{B A}}(5.3 \mathrm{mM})$ after heating at $100{ }^{\circ} \mathrm{C}$ for $3 \mathrm{~min}$. $\mathbf{d}, \mathbf{e}$ Typical photographs of CD-active DMF/water $\left(7 / 3, \mathrm{v} / \mathrm{v} ; 1 \mathrm{~mL}\right.$ ) suspensions of $\mathbf{P B T A}^{\mathbf{B A}}$ ([BTAABA] $=5.3 \mathrm{mM}$ ) and SEM images of their air-dried specimens. The suspensions of (-)-PBTA ${ }^{\mathbf{B A}}$ and (+)-PBTA ${ }^{\mathbf{B A}}$ display mirror-image CD spectra of one another (Fig. 4b). They can be prepared by natural cooling of a clear, hot DMF/water $(7 / 3, \mathrm{v} / \mathrm{v} ; 1 \mathrm{~mL})$ solution of $\mathbf{B T A}^{\mathbf{B A}}(5.3 \mathrm{mM})$ with magnetic rotary stirring at $1200 \mathrm{rpm}$ for 40 min. $\mathbf{f}$ Photograph of a CD-silent precipitate of $\mathbf{P B T A}^{\mathbf{B A}}$ ([BTAA $\left.\left.\mathbf{B}^{\mathbf{B A}}\right]=5.3 \mathrm{mM}\right)$ in $\mathrm{DMF} /$ water $(7 / 3, \mathrm{v} / \mathrm{v} ; 1 \mathrm{~mL})$ and SEM image of its air-dried specimen. This precipitate can be prepared by natural cooling of a clear, hot DMF/water $(7 / 3, \mathrm{v} / \mathrm{v} ; 1 \mathrm{~mL})$ solution of $\mathbf{B T A}^{\mathbf{B A}}(5.3 \mathrm{mM})$ without magnetic rotary stirring 
to the stretching vibrations of hydrogen-bonded amide $\mathrm{NH}$ and $\mathrm{C}=\mathrm{O}$ groups, respectively, were also observed. We found that $\mathbf{B T A}^{\mathbf{B A}}{ }$ is quite soluble in DMF alone $\left(\left[\mathbf{B T A}^{\mathbf{B A}}\right]=5.3 \mathrm{mM}\right)$, where it showed a much sharper absorption band at $290 \mathrm{~nm}$ (Supplementary Fig. 15$)$ than its self-assembled state $\left(\left[\mathbf{B T A} \mathbf{A}^{\mathbf{B A}}\right]=\right.$ $5.3 \mathrm{mM})$ in the gel formed in DMF/water $(7 / 3, \mathrm{v} / \mathrm{v}$, Fig. $2 \mathrm{~b})$. Compared with the DMF solution of $\mathbf{B T A} \mathbf{A}^{\mathbf{B A}}$, the gel was much more luminescent ( 300 times) at $447 \mathrm{~nm}$ upon excitation at $290 \mathrm{~nm}$ (Supplementary Fig. 16), suggesting that the BTA ${ }^{\mathbf{B A}}$ units in the gel network are located in a highly congested environment. Further to note, a DMF/water suspension of PBTA $^{\text {BA }}$ (Fig. 2d/e) was silent in polarized optical microscopy (Supplementary Fig. 17), and a wet sample of PBTA $^{\mathbf{B A}}$ isolated by centrifugation of its suspension showed no X-ray diffraction (Supplementary Fig. 18). These results allow us to conclude that PBTA $^{\mathbf{B A}}$ is a noncrystalline material.

When a clear, hot solution of $\mathbf{B T A}^{\mathbf{B A}}$ (Fig. 2c) in DMF/water $(7 / 3, \mathrm{v} / \mathrm{v})$ was allowed to cool to $25^{\circ} \mathrm{C}$ with continuous rotary stirring, the absorption dissymmetry factor $\left(g_{\text {abs }}\right)$ of the resulting suspension, evaluated from the $C D$ intensity relative to the absorbance at $316 \mathrm{~nm}$, gradually increased with the stirring time and then leveled off in $40 \mathrm{~min}$ at $2.2 \times 10^{-2}$ (Supplementary Fig. 19). We noticed that faster stirring resulted in the emergence of a larger CD intensity at $316 \mathrm{~nm}$ (Supplementary Fig. 20). Rotary stirring of the CD-silent gel did not induce any $C D$ band (Supplementary Fig. 21), indicating that physical agitation such as rotary stirring does not affect the helical handedness of the PBTA $^{\text {BA }}$ nanoribbons once formed. From these observations, we proposed a mechanism for the formation of the $(P)$ - or $(M)$ dominant helical nanoribbons from $\mathbf{B T A}^{\mathbf{B A}}$ as follows: $\mathbf{B T A}^{\mathbf{B A}}$ self-assembles into $(P)$ - and $(M)$-helical short $1 \mathrm{D}$ aggregates (primary nucleation). Such aggregates grow up to longer $(P)$ - and $(M)$-helical nanoribbons, respectively. By physical agitation, the long helical nanoribbons preferentially break up into numerous short aggregates with the same handedness (secondary nucleation). In the resulting nonequilibrated state, once the mirror symmetry of the PBTA $\mathbf{A B}^{\mathbf{B A}}$ nanoribbon is broken as a consequence of stochastic fluctuation, a small chiral bias thus generated can be amplified through repeated cycles of the secondary nucleation, as proposed for the mechanism of chiral amplification via thermal cycling or attrition in conglomerate crystal systems ${ }^{9-11}$. Accordingly, the addition of $(P)$-dominant PBTA $^{\mathbf{B A}}$ as a seed into a DMF/water $(7 / 3, \mathrm{v} / \mathrm{v} ; 1 \mathrm{~mL})$ solution of $\mathbf{B T A}^{\mathbf{B A}}(5.3 \mathrm{mM})$ before rotary stirring always resulted in the formation of a suspension of $(P)$-dominant PBTA $^{\text {BA }}$ with a negative $C D$ band at $316 \mathrm{~nm}$ (Supplementary Fig. 22).

Asymmetric catalysis. Previously, groups of Engberts ${ }^{56,57}$ and Roelfes ${ }^{25,27}$ reported highly enantioselective $\mathrm{Cu}^{2+}$-catalyzed Diels-Alder reactions between aza-chalcone and cyclopentadiene using amino acids and DNA as the chiral mediators, respectively. Inspired by these successful examples, we chose the same reaction to investigate a possibility of mirror symmetry-broken PBTA $^{\mathbf{B A}}$ nanoribbons as a chiral ligand. Typically, $\mathrm{Cu}^{2+}$-coordinated $(-)-$ PBTA $^{\mathbf{B A}}$ or $(+)-$ PBTA $^{\mathbf{B A}} \quad\left(\left[\mathrm{Cu}^{2+}\right] /\left[\mathbf{B T A}^{\mathbf{B A}}\right]=1.0 \%\right)$, employed as a catalyst for the Diels-Alder reaction (Fig. 3a), was prepared by mixing an aqueous solution of $\mathrm{Cu}\left(\mathrm{NO}_{3}\right)_{2}(4.1 \mathrm{mM}$, $13 \mu \mathrm{L})$ and a DMF/water $(7 / 3, \mathrm{v} / \mathrm{v} ; 1 \mathrm{~mL})$ suspension of $(-)-$ PBTA $^{\mathbf{B A}}$ or $(+)-$ PBTA $^{\mathbf{B A}}\left(\left[\right.\right.$ BTA $\left.^{\mathbf{B A}}\right]=5.3 \mathrm{mM}$, Fig. $\left.2 \mathrm{~d}, \mathrm{e}\right)$ at $25^{\circ} \mathrm{C}$ for $1 \mathrm{~h}$. The luminescence of $\mathbf{P B T A}{ }^{\mathbf{B A}}$ was quenched upon being mixed with $\mathrm{Cu}\left(\mathrm{NO}_{3}\right)_{2}$ (Supplementary Fig. 23), suggesting that PBTA ${ }^{\mathbf{B A}}$ accommodates $\mathrm{Cu}^{2+}$ presumably at the peripheral carboxylate units. As observed by SEM and CD spectroscopy, PBTA $^{\mathbf{B A}}$ maintained its helical nanoribbon structure even after being mixed with $\mathrm{Cu}^{2+}$ (Supplementary Fig. 24). For the
Diels-Alder reaction, an acetonitrile $(40 \mu \mathrm{L})$ solution of azachalcone $(1 \mathrm{mg})$ and freshly distilled cyclopentadiene $(40 \mu \mathrm{L})$ were successively added to a DMF/water $(7 / 3, \mathrm{v} / \mathrm{v})$ suspension of $(-)-\mathbf{P B T A}^{\mathbf{B A}} / \mathrm{Cu}^{2+}$ or $(+)-\mathbf{P B T A} \mathbf{B A}^{\mathbf{B A}} / \mathrm{Cu}^{2+}$, and the mixture was stirred at $25^{\circ} \mathrm{C}$. After $36 \mathrm{~h}$, the reaction mixture was extracted with ethyl acetate $(0.5 \mathrm{~mL})$, and an organic extract, after being evaporated to dryness, was subjected to chiral high performance liquid chromatography (HPLC), where the endo isomer was revealed to be the major product ( $\sim 90 \%$, conversion: $>99 \%$, turnover number: $\sim 90$, Fig. 3b, c). Of particular interest, (-)-PBTA ${ }^{\mathbf{B A}} / \mathrm{Cu}^{2+}$ (Fig. 2d) and (+)-PBTA ${ }^{\mathbf{B A}} / \mathrm{Cu}^{2+}$ (Fig. 2e) preferentially afforded the enantiomers of $(S)$-endo (Fig. 3b) and (R)-endo (Fig. 3c), respectively. We conducted a total of 76 runs of the Diels-Alder reaction using 76 different batches of $(-)-\mathbf{P B T A}^{\mathbf{B A}} / \mathrm{Cu}^{2+}$ and (+)-PBTA ${ }^{\mathbf{B A}} / \mathrm{Cu}^{2+}$ under the conditions described above and confirmed that this stereochemical correlation between the catalyst and product was perfectly reproduced (Fig. 3d and Supplementary Table 3). However, there is no clear correlation between the CD intensities of the catalyst and enantiomeric excess values of the product (Supplementary Fig. 25). We confirmed that the nanoribbons of PBTA ${ }^{\mathbf{B A}}$ after the reaction were shorter than those before the reaction (Supplementary Fig. 26). However, the ee value of the endo isomer did not substantially change with the reaction time (Supplementary Fig. 27). In sharp contrast, repeated usage of the catalyst, isolated each time via centrifugation from the reaction mixture, resulted in lowering of the enantiomeric excess of the product (Supplementary Table 4). Meanwhile, CD-silent PBTA ${ }^{\mathbf{B A}} / \mathrm{Cu}^{2+}$ nanoribbons (Fig. 2b) also catalyzed the Diels-Alder reaction. However, as expected, the reaction was not enantioselective (Supplementary Fig. 28), as in the case where PBTA ${ }^{\mathbf{B A}}$ was not employed for the $\mathrm{Cu}^{2+}$-catalyzed Diels-Alder reaction (Supplementary Fig. 29).

We attempted to optimize the conditions for the Diels-Alder reaction. First, we varied $\left[\mathrm{Cu}^{2+}\right]$ versus $\left[\mathbf{B T A}^{\mathbf{B A}}\right]$ and found that the $e e$ value of the endo isomer reached a maximum at $\left[\mathrm{Cu}^{2+}\right] /$ $\left[\mathbf{B T A}^{\mathbf{B A}}\right]=1.0 \%$ (Fig. 3e). When $\left[\mathrm{Cu}^{2+}\right] /\left[\mathbf{B T A}^{\mathbf{B A}}\right]$ exceeded $1.0 \%$, the ee value of the endo isomer dropped, most likely because of a possible increase in the amount of $\mathrm{Cu}^{2+}$ unanchored to the helical PBTA ${ }^{\mathbf{B A}}$ nanoribbons. A strong base such as $\mathrm{NaOH}$ is expected to deprotonate the benzoic acid groups of $\mathbf{P B T A}^{\mathbf{B A}}$ to promote the anchoring of $\mathrm{Cu}^{2+}$ onto the helical PBTA ${ }^{\mathbf{B A}}$ nanoribbons ${ }^{36}$. In fact, as shown in Fig. $3 \mathrm{f}$, the addition of $\mathrm{NaOH}$ to the reaction system resulted in a considerable enhancement of the enantioselectivity of the reaction, where an average $e e$ value of $>40 \%$ was realized at $[\mathrm{NaOH}] /\left[\mathbf{B T A}^{\mathbf{B A}}\right]=$ $50 \%$ with the highest $e e$ value of $46 \%$ (major/minor enantiomers $=73 / 27$; Supplementary Fig. 30).

Chirality transfer via electrostatic interaction. We explored the stereochemical environment of the cation-binding sites in the helical PBTA ${ }^{\mathbf{B A}}$ nanoribbon by using a cationic fluorescent dye, methylene blue (MB, Fig. 4a). When an aqueous solution of $\mathrm{MB}$ $(21.2 \mathrm{mM}, 50 \mu \mathrm{L})$ was added to a DMF/water $(7 / 3, \mathrm{v} / \mathrm{v} ; 1 \mathrm{~mL})$ suspension of mirror symmetry-broken (-)-PBTA ${ }^{\mathbf{B A}}\left(\left[\mathbf{B T A}^{\mathbf{B A}}\right]\right.$ $\left.=5.3 \mathrm{mM},[\mathrm{MB}] /\left[\mathbf{B T A}^{\mathbf{B A}}\right]=20 \%\right)$, a broad positive $\mathrm{CD}$ band at 570-700 nm emerged (Fig. 4d and Supplementary Fig. 31). When $(+)-$ PBTA $^{\mathbf{B A}}\left(\left[\mathbf{B T A}^{\mathbf{B A}}\right]=5.3 \mathrm{mM}\right)$ was employed instead of (-)-PBTA for mixing with $\mathrm{MB}$, the sign of the $\mathrm{CD}$ band at 570-700 nm was negative (Fig. 4d and Supplementary Fig. 31). Considering that $\mathrm{MB}$ has an absorption band at $570-700 \mathrm{~nm}$ (Supplementary Fig. 31), the CD bands in this region are assignable to $\mathrm{MB}$, which is supposedly located in a chiral environment by being bound to the helical nanoribbon of PBTA ${ }^{\mathbf{B A}}$ at its carboxylate units (Fig. $4 a)^{58,59}$. Note that mirror symmetrybroken (-)-PBTA ${ }^{\mathbf{B A}} / \mathrm{MB}$ and (+)-PBTA $\mathbf{B A}^{\mathbf{B A}} / \mathrm{MB}$ displayed 
a<smiles>O=C(/C=C/c1ccccc1)c1ccccn1</smiles>
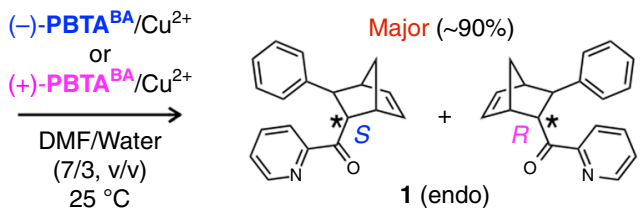

b

Chiral HPLC (see no. 1 in d)

C Chiral HPLC (see no. 34 in d)
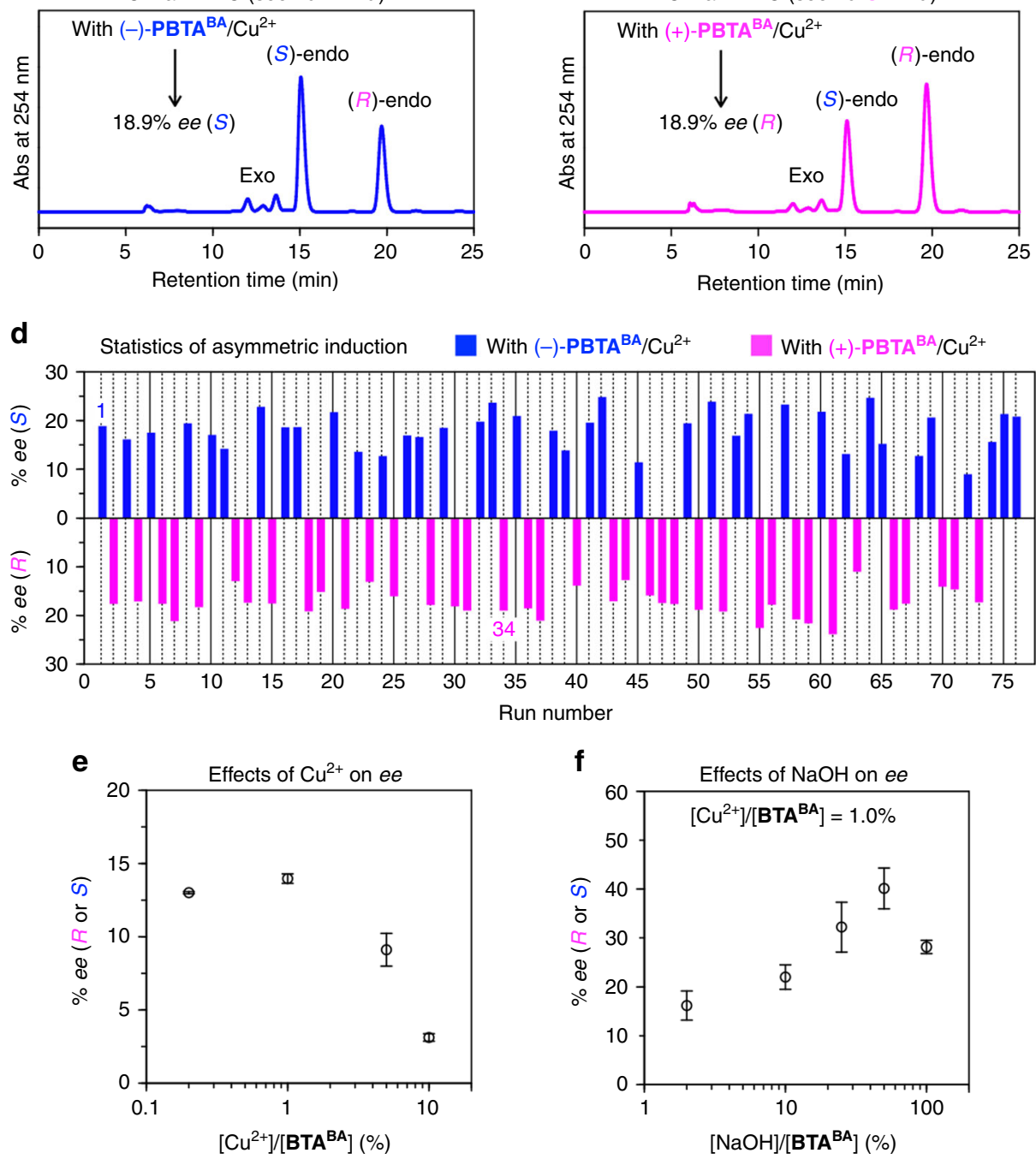

Fig. 3 Enantioselective Diels-Alder reaction catalyzed by $\mathrm{Cu}^{2+}$-coordinated $\mathbf{P B T A} \mathbf{A}^{\mathbf{B A}}$ nanoribbons. a Diels-Alder reaction between aza-chalcone and cyclopentadiene catalyzed by (-)-PBTA $\mathbf{B A}^{\mathbf{B A}} \mathrm{Cu}^{2+}$ or $(+)-\mathbf{P B T A}^{\mathbf{B A}} / \mathrm{Cu}^{2+}$, which preferentially affords the endo isomer ( $\left.90 \%\right)$. $\mathbf{b}, \mathbf{c}$ Chiral HPLC traces of the reaction mixtures in run No. $1(\mathbf{b})$ and $34(\mathbf{c})$ in $(\mathbf{d})$. Symbols ee $(S)$ and ee $(R)$ represent enantiomeric excess (ee) values of the endo isomers with the dominancy of their $(S)$-endo and $(R)$-endo enantiomers, respectively. $\mathbf{d}$ Statistical ee values of the endo isomers obtained from a total of 76 runs of the Diels-Alder reaction catalyzed at $25^{\circ} \mathrm{C}$ by 76 different batches of $(-)-\mathbf{P B T A}^{\mathbf{B A}} / \mathrm{Cu}^{2+}$ and $(+)-\mathbf{P B T A} \mathbf{B A}^{\mathbf{B A}} / \mathrm{Cu}^{2+}$ at $\left[\mathrm{Cu}^{2+}\right] /\left[\mathbf{B T A}^{\mathbf{B A}}\right]=1.0 \%$. Blue and pinkcolored bars represent the ee values of the endo isomers obtained when (-)-PBTA ${ }^{\mathbf{B A}} / \mathbf{C u}^{2+}$ and $(+)-\mathbf{P B T A} \mathbf{A B}^{\mathbf{B A}} / \mathrm{Cu}^{2+}$, respectively, are used as the catalysts. e Effects of $\left[\mathrm{Cu}^{2+}\right] /\left[\mathbf{B T A}^{\mathbf{B A}}\right]$ on the ee values of the endo isomers obtained at $25^{\circ} \mathrm{C}$ with $(-)-\mathbf{P B T A} \mathbf{A}^{\mathbf{B A}} / \mathrm{Cu}^{2+}$ or $(+)-\mathbf{P B T A}^{\mathbf{B A}} / \mathrm{Cu}^{2+}$. $\mathbf{f}$ Effects of $[\mathrm{NaOH}] /\left[\mathbf{B T A}^{\mathbf{B A}}\right]$ on the ee values of the endo isomers obtained at $25^{\circ} \mathrm{C}$ with $(-)-\mathbf{P B T A} \mathbf{A}^{\mathbf{B A}} / \mathrm{Cu}^{2+}$ or $(+)-\mathbf{P B T A}^{\mathbf{B A}} / \mathrm{Cu}^{2+}$ at $\left[\mathrm{Cu}^{2+}\right] /\left[\mathbf{B T A} \mathbf{B A}^{\mathbf{B A}}\right]=1.0 \%$, where $\mathrm{NaOH}$ was added to the suspensions of $(-)-\mathbf{P B T A} \mathbf{B A}^{\mathbf{B A}}$ or $(+)-\mathbf{P B T A} \mathbf{B A}$ prior to the addition of $\mathrm{Cu}\left(\mathrm{NO}_{3}\right)_{2}$. Error bars represent the standard deviation. Source data are provided as a Source Data file

positive and negative circularly polarized luminescence (CPL) bands at $600-800 \mathrm{~nm}$, respectively $\left(\lambda_{\text {ext }}=550 \mathrm{~nm}\right.$, Fig. $4 \mathrm{e}$ and Supplementary Fig. 32b). These CPL bands are obviously different from those observed for (-)-PBTA ${ }^{\mathbf{B A}}$ and $(+)-\mathbf{P B T A}^{\mathbf{B A}}$ $\left(400-600 \mathrm{~nm}, \lambda_{\text {ext }}=290 \mathrm{~nm}\right.$; Fig. $4 \mathrm{c}$ and Supplementary Fig. 32a) and therefore are assignable to $\mathrm{MB}$. The luminescence dissymmetry factors $\left(g_{\text {lum }}\right)$ of $(-)-\mathbf{P B T A}^{\mathbf{B A}} / \mathrm{MB}$ and $(+)-\mathbf{P B T A}^{\mathbf{B A}} /$ $\mathrm{MB}$, evaluated from their CPL intensities relative to the fluorescence intensities at $700 \mathrm{~nm}$, were $\sim 10^{-2}$, which are much larger than those commonly observed for chiral organic compounds $\left(10^{-5}-10^{-2}\right)^{60}$. This result again indicates unambiguously that MB is localized in a chiral environment provided by $(-)-\mathbf{P B T A}^{\mathbf{B A}}$ or $(+)-\mathbf{P B T A}^{\mathbf{B A}}$.

\section{Discussion}

All of these results allow us to conclude that a mirror symmetrybroken helical supramolecular nanoribbon composed of an 
a

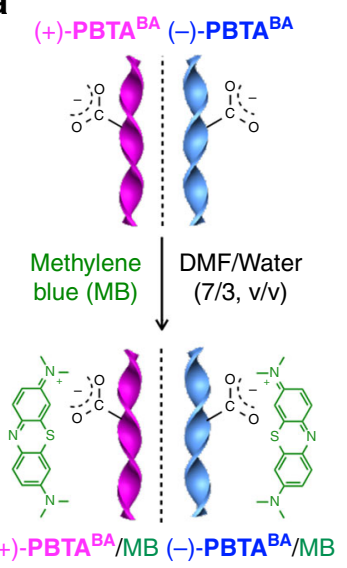

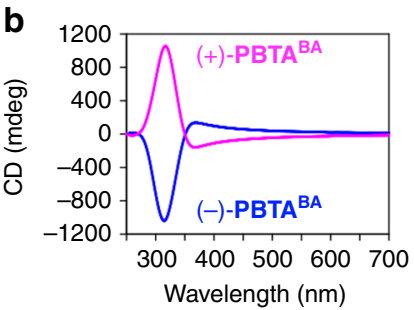
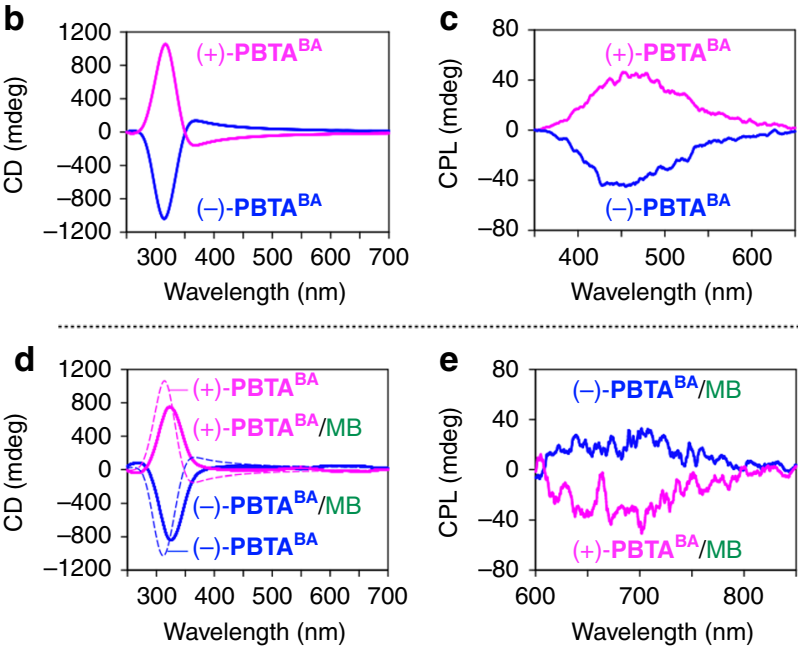

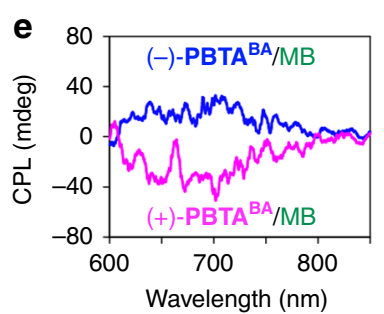

Fig. 4 Chiral environment of the cation-binding sites in $\mathbf{P B T A}^{\mathbf{B A}}$ nanoribbons. a Schematic representation of the binding of methylene blue (MB) onto PBTA $^{\text {BA }}$ at its carboxylate units via an electrostatic interaction. $\mathbf{b}, \mathbf{c}$ Circular dichroism $(C D, \mathbf{b})$ and circularly polarized luminescence $\left(C P L, \lambda_{\text {ext }}=290 \mathrm{~nm}\right.$, c) spectra at $25^{\circ} \mathrm{C}$ of DMF/water (7/3, v/v; $1 \mathrm{~mL}$ ) suspensions of (-)-PBTABA (blue) and (+)-PBTABA (pink) ([BTA $\left.\mathbf{A}^{\mathbf{B A}}\right]=5.3 \mathrm{mM}$, Fig. $\left.2 \mathrm{~d}, \mathrm{e}\right)$. d, e CD (d) and CPL $\left(\lambda_{\text {ext }}=550 \mathrm{~nm}, \mathbf{e}\right)$ spectra at $25^{\circ} \mathrm{C}$ of DMF/water $(7 / 3, \mathrm{v} / \mathrm{v} ; 1 \mathrm{~mL})$ suspensions of $(-)$-PBTA ${ }^{\mathbf{B A}}$ (blue) and (+)-PBTA ${ }^{\mathbf{B A}}$ (pink) $\left(\left[\mathbf{B T A}^{\mathbf{B A}}\right]=\right.$ $5.3 \mathrm{mM})$ containing $\mathrm{MB}\left([\mathrm{MB}] /\left[\mathbf{B T A}^{\mathbf{B A}}\right]=20 \%\right)$. Source data are provided as a Source Data file

achiral monomer can serve as a chiral ligand for mediating enantioselective catalysis. Although the noncrystalline nature of PBTA $^{\text {BA }}$ (Supplementary Figs. 17 and 18) does not permit elucidation of its molecular packing, the successful CPL observation from $\mathrm{MB}$ in Fig. 4e suggests that the catalytic site having a $\mathrm{Cu}^{2+}$ ion is chiral. As for the modest enantioselectivity of the reaction, we assume that this is due to the helical nanoribbon structure, which is formed by rolling up of a rather planer, sheet structure. It is likely that the catalytic sites located on such a gently curved surface cannot efficiently bias the stereochemical course of the reaction. We also noted that the mirror symmetry-broken helical nanoribbons of $\mathbf{B T A}^{\mathbf{B A}}$ display intense electronic $\mathrm{CD}$ $\left(\Delta \varepsilon=\sim 570 \mathrm{~L} \mathrm{~mol}^{-1} \mathrm{~cm}^{-1}\right)$. This is very rare, but some helicene nanoribbons are known to show such an amplified chiroptical behavior $^{61}$. The next grand challenge to tackle is how one can obtain a helical catalyst with a preferred handedness by mirror symmetry breaking. Together with our achievement, the design of mirror symmetry breaking may revolutionize the manufacturing process of chiral compounds having many potential applications.

\section{Methods}

Materials. Unless otherwise noted, reagents and solvents were used as received from commercial sources without further purification. Benzene-1,3,5-tricarbonyl trichloride was purchased from Alfa Aesar. Ethyl 4-aminobenzoate was purchased from Adamas. Copper (II) nitrate $\left(\mathrm{Cu}\left(\mathrm{NO}_{3}\right)_{2}\right)$ was purchased from J\&K Scientific. Methylene blue was purchased from Tokyo Chemical Industry (TCI). Dicyclopentadiene was purchased from Acros Organics. Cyclopentadiene was prepared by distillation of commercial dicyclopentadiene at $180^{\circ} \mathrm{C}$. (E)-3-Phenyl-1-(pyridin-2-yl)prop-2-en-1-one (aza-chalcone) was prepared according to a previously reported method ${ }^{56}$.

Characterization. ${ }^{1} \mathrm{H}$ and ${ }^{13} \mathrm{C}$ nuclear magnetic resonance (NMR) spectra were recorded on a JEOL model ALPHA-500 spectrometer in which the chemical shifts for ${ }^{1} \mathrm{H}$ NMR spectroscopy were determined with respect to a non-deuterated solvent residue, $\mathrm{CHD}_{2}\left(\mathrm{CD}_{3}\right) \mathrm{SO}(\delta 2.50 \mathrm{ppm})$, and those for ${ }^{13} \mathrm{C}$ NMR spectroscopy were determined with respect to DMSO ( $\delta 39.52 \mathrm{ppm})$. Matrix-assisted laser desorption/ionization time-of-flight mass (MALDI-TOF MS) spectrometry was performed using $\alpha$-cyano-4-hydroxycinnamic acid (CHCA) as a matrix using a Bruker Daltonics Autoflex ${ }^{\mathrm{TM}}$ Speed MALDI-TOF/TOF spectrometer. Electronic absorption spectra were recorded in sandwich-type quartz cuvettes with an optical path length of $0.1 \mathrm{~mm}$ using a JASCO UV-550 spectrometer. CD and LD spectra were recorded in sandwich-type quartz cuvettes with an optical path length of $0.1 \mathrm{~mm}$ using a JASCO J-810 spectrometer. Circularly polarized luminescence (CPL) spectra were recorded in sandwich-type quartz cuvettes with an optical path length of $0.1 \mathrm{~mm}$ using a JASCO CPL-200 spectrometer. Fluorescence spectra were recorded using a
Hitachi F-4500 spectrophotometer. FT-IR spectra were recorded using a JASCO model FT/IR-6100Plus FT-IR spectrometer. SEM was performed using a Hitachi S$4800 \mathrm{FE}-\mathrm{SEM}$ with an accelerating voltage of $10 \mathrm{kV}$. X-ray diffraction (XRD) analysis was performed on a Rigaku D/Max-2500 X-ray diffractometer (Japan) with $\mathrm{Cu}$ Ka radiation $(\lambda=1.5406 \AA)$, which was operated at a voltage of $40 \mathrm{kV}$ and a current of $200 \mathrm{~mA}$. Polarized optical microscopy (POM) was performed using a Leica DM2700 upright materials microscope. Chiral HPLC was performed using a Waters 1525 HPLC system with a Daicel Chiralcel ${ }^{\circledR}$ OD-H column.

\section{Data availability}

The source data underlying Fig. 3b-f and 4b-e and Supplementary Figs. 1-11, 12a, e, h, $14-16,18-21,23,24 b, 25$ and $27-32$ are provided as a Source Data file, which is available via figshare (DOI: $10.6084 / \mathrm{m} 9$.figshare.8940404).

Received: 12 January 2019 Accepted: 25 July 2019

Published online: 04 September 2019

\section{References}

1. Mason, S. F. Origins of biomolecular handedness. Nature 311, 19-23 (1984).

2. Bada, J. L. Biomolecules-origins of homochirality. Nature 374, 594-595 (1995).

3. Penzien, K. G. \& Schmidt, M. J. Reactions in chiral crystals: an absolute asymmetric synthesis. Angew. Chem. Int. Ed. 8, 608-609 (1969).

4. Green, B. S. \& Heller, L. Mechanism for the autocatalytic formation of optically active compounds under abiotic conditions. Science 185, 525-527 (1974).

5. Green, B. S., Lahav, M. \& Rabinovich, D. Asymmetric synthesis via reactions in chiral crystals. Acc. Chem. Res. 12, 191-197 (1979).

6. Barron, L. D. Symmetry and molecular chirality. Chem. Soc. Rev. 15, 189-223 (1986).

7. Feringa, B. L. \& van Delden, R. A. Absolute asymmetric synthesis: the origin, control, and amplification of chirality. Angew. Chem. Int. Ed. 38, 3418-3438 (1999).

8. Cintas, P. \& Viedma, C. On the physical basis of asymmetry and homochirality. Chirality 24, 894-908 (2012).

9. Kondepudi, D. K., Kaufman, R. J. \& Singh, N. Chiral symmetry breaking in sodium chlorate crystallization. Science 250, 975-976 (1990).

10. McBride, J. M. \& Carter, R. L. Spontaneous resolution by stirred crystallization. Angew. Chem. Int. Ed. 30, 293-295 (1991).

11. Kondepudi, D. K., Bullock, K. L., Digits, J. A. \& Yarborough, P. D. Stirring rate as a critical parameter in chiral-symmetry breaking crystallization. J. Am. Chem. Soc. 117, 401-404 (1995).

12. Viedma, C. Chiral symmetry breaking during crystallization: complete chiral purity induced by nonlinear autocatalysis and recycling. Phys. Rev. Lett. 94, 065504 (2005). 
13. Noorduin, W. L. et al. Emergence of a single solid chiral state from a nearly racemic amino acid derivative. J. Am. Chem. Soc. 130, 1158-1159 (2008).

14. Viedma, C., Ortiz, J. E., Torres, Td, Izumi, T. \& Blackmond, D. G. Evolution of solid phase homochirality for a proteinogenic amino acid. J. Am. Chem. Soc. 130, 15274-15275 (2008).

15. Noorduin, W. L. et al. Complete deracemization by attrition-enhanced ostwald ripening elucidated. Angew. Chem. Int. Ed. 47, 6445-6447 (2008).

16. Hein, J. E., Huynh Cao, B., Viedma, C., Kellogg, R. M. \& Blackmond, D. G. Pasteur's tweezers revisited: on the mechanism of attrition-enhanced deracemization and resolution of chiral conglomerate solids. J. Am. Chem. Soc. 134, 12629-12636 (2012).

17. Sogutoglu, L.-C., Steendam, R. R. E., Meekes, H., Vlieg, E. \& Rutjes, F. P. J. T. Viedma ripening: a reliable crystallisation method to reach single chirality. Chem. Soc. Rev. 44, 6723-6732 (2015).

18. Steendam, R. R. E. et al. Emergence of single-molecular chirality from achiral reactants. Nat. Commun. 5, 5543 (2014).

19. Blackmond, D. G. "Chiral amnesia" as a driving force for solid-phase homochirality. Chem. Eur. J. 13, 3290-3295 (2007).

20. Blackmond, D. G. Response to "comments on a possible transition to solidphase homochirality". Chem. Eur. J. 13, 10306-10311 (2007).

21. Tsogoeva, S. B., Wei, S., Freund, M. \& Mauksch, M. Generation of highly enantioenriched crystalline products in reversible asymmetric reactions with racemic or achiral catalysts. Angew. Chem. Int. Ed. 48, 590-594 (2009).

22. Wei, S., Mauksch, M. \& Tsogoeva, S. B. Autocatalytic enantiomerisation at the crystal surface in deracemisation of scalemic conglomerates. Chem. Eur. J. 15, 10255-10262 (2009).

23. Kawasaki, T., Suzuki, K., Hakoda, Y. \& Soai, K. Achiral nucleobase cytosine acts as an origin of homochirality of biomolecules in conjunction with asymmetric autocatalysis. Angew. Chem. Int. Ed. 47, 496-499 (2008).

24. Soai, K., Kawasaki, T. \& Matsumoto, A. Asymmetric autocatalysis of pyrimidyl alkanol and its application to the study on the origin of homochirality. Acc. Chem. Res. 47, 3643-3654 (2014).

25. Roelfes, G. \& Feringa, B. L. DNA-based asymmetric catalysis. Angew. Chem. Int. Ed. 44, 3230-3232 (2005).

26. Boersma, A. J. et al. Catalytic enantioselective syn hydration of enones in water using a DNA-based catalyst. Nat. Chem. 2, 991-995 (2010).

27. Boersma, A. J., Megens, R. P., Feringa, B. L. \& Roelfes, G. DNA-based asymmetric catalysis. Chem. Soc. Rev. 39, 2083-2092 (2010).

28. Reggelin, M., Schultz, M. \& Holbach, M. Helical chiral polymers without additional stereogenic units: a new class of ligands in asymmetric catalysis. Angew. Chem. Int. Ed. 41, 1614-1617 (2002).

29. Yamamoto, T. \& Suginome, M. Helical poly(quinoxaline-2,3-diyl)s bearing metal-binding sites as polymer-based chiral ligands for asymmetric catalysis. Angew. Chem. Int. Ed. 48, 539-542 (2009).

30. Yamamoto, T., Yamada, T., Nagata, Y. \& Suginome, M. High-molecular-weight polyquinoxaline-based helically chiral phosphine (PQXphos) as chiralityswitchable, reusable, and highly enantioselective monodentate ligand in catalytic asymmetric hydrosilylation of styrenes. J. Am. Chem. Soc. 132, 7899-7901 (2010)

31. Yamamoto, T., Akai, Y., Nagata, Y. \& Suginome, M. Highly enantioselective synthesis of axially chiral biarylphosphonates: asymmetric Suzuki-Miyaura coupling using high-molecular-weight, helically chiral polyquinoxaline-based phosphines. Angew. Chem. Int. Ed. 50, 8844-8847 (2011).

32. Akai, Y., Yamamoto, T., Nagata, Y., Ohmura, T. \& Suginome, M. Enhanced catalyst activity and enantioselectivity with chirality-switchable polymer ligand PQXphos in Pd-catalyzed asymmetric silaborative cleavage of mesomethylenecyclopropanes. J. Am. Chem. Soc. 134, 11092-11095 (2012).

33. Yamamoto, T., Akai, Y. \& Suginome, M. Chiral palladacycle catalysts generated on a single-handed helical polymer skeleton for asymmetric arylative ring opening of 1,4-epoxy-1,4-dihydronaphthalene. Angew. Chem. Int. Ed. 53, 12785-12788 (2014).

34. Ke, Y.-Z., Nagata, Y., Yamada, T. \& Suginome, M. Majority-rules-type helical poly(quinoxaline-2,3-diyl)s as highly efficient chirality-amplification systems for asymmetric catalysis. Angew. Chem. Int. Ed. 54, 9333-9337 (2015).

35. Iida, H., Iwahana, S., Mizoguchi, T. \& Yashima, E. Main-chain optically active riboflavin polymer for asymmetric catalysis and its vapochromic behavior. $J$. Am. Chem. Soc. 134, 15103-15113 (2012).

36. Jiang, J., Meng, Y., Zhang, L. \& Liu, M. Self-assembled single-walled metalhelical nanotube (M-HN): creation of efficient supramolecular catalysts for asymmetric reaction. J. Am. Chem. Soc. 138, 15629-15635 (2016)

37. Raynal, M., Portier, F., van Leeuwen, P. W. N. M. \& Bouteiller, L. Tunable asymmetric catalysis through ligand stacking in chiral rigid rods. J. Am. Chem. Soc. 135, 17687-17690 (2013).

38. Desmarchelier, A. et al. Correlation between the selectivity and the structure of an asymmetric catalyst built on a chirally amplified supramolecular helical scaffold. J. Am. Chem. Soc. 138, 4908-4916 (2016).
39. Zimbron, J. M. et al. Real-time control of the enantioselectivity of a supramolecular catalyst allows selecting the configuration of consecutively formed stereogenic centers. Angew. Chem. Int. Ed. 56, 14016-14019 (2017).

40. Huerta, E. et al. Triggering activity of catalytic rod-like supramolecular polymers. Chem. Eur. J. 21, 3682-3690 (2015)

41. Shen, Z., Wang, T. \& Liu, M. Macroscopic chirality of supramolecular gels formed from achiral tris(ethyl cinnamate) benzene-1,3,5-tricarboxamides. Angew. Chem. Int. Ed. 53, 13424-13428 (2014).

42. Shen, Z., Wang, T., Shi, L., Tang, Z. \& Liu, M. Strong circularly polarized luminescence from the supramolecular gels of an achiral gelator: tunable intensity and handedness. Chem. Sci. 6, 4267-4272 (2015).

43. DeRossi, U., Dahne, S., Meskers, S. C. J. \& Dekkers, H. Spontaneous formation of chirality in J-aggregates showing davydov splitting. Angew. Chem. Int. Ed. 35, 760-763 (1996)

44. Ribo, J. M., Crusats, J., Sagues, F., Claret, J. \& Rubires, R. Chiral sign induction by vortices during the formation of mesophases in stirred solutions. Science 292, 2063-2066 (2001).

45. Yuan, J. \& Liu, M. Chiral molecular assemblies from a novel achiral amphiphilic 2-(heptadecyl) naphtha[2,3]imidazole through interfacial coordination. J. Am. Chem. Soc. 125, 5051-5056 (2003).

46. Zhang, S., Yang, S., Lan, J., Yang, S. \& You, J. Helical nonracemic tubular coordination polymer gelators from simple achiral molecules. Chem. Commun. 44, 6170-6172 (2008)

47. Micali, N. et al. Selection of supramolecular chirality by application of rotational and magnetic forces. Nat. Chem. 4, 201-207 (2012).

48. Azeroual, S. et al. Mirror symmetry breaking and chiral amplification in foldamer-based supramolecular helical aggregates. Chem. Commun. 48, 2292-2294 (2012).

49. Stals, P. J. M. et al. Symmetry breaking in the self-assembly of partially fluorinated benzene-1,3,5-tricarboxamides. Angew. Chem. Int. Ed. 51, 11297-11301 (2012)

50. Wang, Y. et al. Hydrogen-bonded supercoil self-assembly from achiral molecular components with light-driven supramolecular chirality. J. Mater. Chem. C 2, 6402-6409 (2014)

51. Li, Y., Wong, K. M.-C., Wong, H.-L. \& Yam, V. W.-W. Helical self-assembly and photopolymerization properties of achiral amphiphilic platinum(II) diacetylene complexes of tridentate 2,6-bis(1-alkylpyrazol-3-yl)pyridines. ACS Appl. Mat. Interfaces 8, 17445-17453 (2016).

52. Hu, J. et al. Chiral assemblies from an achiral pyridinium-tailored anthracene Chem. Eur. J. 23, 1422-1426 (2017).

53. Karunakaran, S. C., Cafferty, B. J., Weigert-Munoz, A., Schuster, G. B. \& Hud N. V. Spontaneous symmetry breaking in the formation of supramolecular polymers: implications for the origin of biological homochirality. Angew. Chem. Int. Ed. 58, 1453-1457 (2019).

54. Perez-Garcia, L. \& Amabilino, D. B. Spontaneous resolution, whence and whither: from enantiomorphic solids to chiral liquid crystals, monolayers and macro- and supra-molecular polymers and assemblies. Chem. Soc. Rev. 36, 941-967 (2007).

55. Bernet, A., Albuquerque, R. Q., Behr, M., Hoffmann, S. T. \& Schmidt, H. W. Formation of a supramolecular chromophore: a spectroscopic and theoretical study. Soft Matter 8, 66-69 (2012).

56. Otto, S., Boccaletti, G. \& Engberts, J. B. F. N. A chiral Lewis-acid-catalyzed Diels-Alder reaction. Water-enhanced enantioselectivity. J. Am. Chem. Soc. 120, 4238-4239 (1998).

57. Otto, S. \& Engberts, J. B. F. N. A systematic study of ligand effects on a Lewisacid-catalyzed Diels-Alder reaction in water. Water-enhanced enantioselectivity. J. Am. Chem. Soc. 121, 6798-6806 (1999).

58. Matthews, J. R., Tuncel, D., Jacobs, R. M. J., Bain, C. D. \& Anderson, H. L. Surfaces designed for charge reversal. J. Am. Chem. Soc. 125, 6428-6433 (2003).

59. Zhang, B., Yue, L., Wang, Y., Yang, Y. \& Wu, L. A novel single-side azobenzene-grafted Anderson-type polyoxometalate for recognition-induced chiral migration. Chem. Commun. 50, 10823-10826 (2014).

60. Sánchez-Carnerero, E. M. et al. Circularly polarized luminescence by visiblelight absorption in a chiral O-BODIPY dye: unprecedented design of CPL organic molecules from achiral chromophores. J. Am. Chem. Soc. 136, 3346-3349 (2014)

61. Schuster, N. J. et al. A helicene nanoribbon with greatly amplified chirality. $J$ Am. Chem. Soc. 140, 6235-6239 (2018).

\section{Acknowledgements}

This work was jointly supported by the Basic Research Development Program of China (2013CB834504), the National Natural Science Foundation of China (91427302), the "Strategic Priority Research Program" of the Chinese Academy of Sciences (XDB12020200) and the Key Research Program of Frontier Sciences, Chinese Academy of Sciences (QYZDJ-SSW-SLH044) to M.L. This work was also supported by the JSPS Grant-in-Aid for Scientific Research (S) (18H05260) on "Innovative Functional Materials based on Multi-Scale Interfacial Molecular Science" to T.A. 


\section{Author contributions}

Z.S., T.A., and M.L. conceived the project. Z.S., Y.S., T.W., J.J., K.O., T.A., and M.L. designed experiments. Z.S., Y.S., J.J., Y.M., and Y.J. performed and interpreted the experiments. Z.S., T.W., K.O., T.A., and M.L. contributed to the writing and editing of the paper. All the authors discussed the results and commented on the paper. Z.S., T.W., K.O., T.A., and M.L. directed the research.

\section{Additional information}

Supplementary Information accompanies this paper at https://doi.org/10.1038/s41467019-11840-3.

Competing interests: The authors declare no competing interests.

Reprints and permission information is available online at http://npg.nature.com/ reprintsandpermissions/

Peer review information: Nature Communications thanks Louis Cuccia, and the other, anonymous, reviewer(s) for their contribution to the peer review of this work. Peer reviewer reports are available.
Publisher's note: Springer Nature remains neutral with regard to jurisdictional claims in published maps and institutional affiliations.

(c) (i) Open Access This article is licensed under a Creative Commons Attribution 4.0 International License, which permits use, sharing, adaptation, distribution and reproduction in any medium or format, as long as you give appropriate credit to the original author(s) and the source, provide a link to the Creative Commons license, and indicate if changes were made. The images or other third party material in this article are included in the article's Creative Commons license, unless indicated otherwise in a credit line to the material. If material is not included in the article's Creative Commons license and your intended use is not permitted by statutory regulation or exceeds the permitted use, you will need to obtain permission directly from the copyright holder. To view a copy of this license, visit http://creativecommons.org/ licenses/by/4.0/.

(c) The Author(s) 2019 